\title{
LY294002 suppresses the malignant phenotype and sensitizes osteosarcoma cells to pirarubicin chemotherapy
}

\author{
XIN HUA LONG ${ }^{1 *}$, ZHEN HAO ZHONG ${ }^{2 *}$, AI FEN PENG $^{3}$, LIANG BO ZHU ${ }^{1}$, \\ HENG WANG ${ }^{1}$, GUO MEI ZHANG ${ }^{4}$ and ZHI LI LIU ${ }^{1}$ \\ ${ }^{1}$ Department of Orthopedics, First Affiliated Hospital of Nanchang University; ${ }^{2}$ Graduate School of Nanchang University; \\ ${ }^{3}$ Jiangxi University of Traditional Chinese Medicine; ${ }^{4}$ Department of Orthopedics, \\ Second Affiliated Hospital of Nanchang University, Nanchang, Jiangxi 330006, P.R. China
}

Received November 30, 2013; Accepted May 19, 2014

DOI: $10.3892 / \mathrm{mmr} .2014 .2617$

\begin{abstract}
Pirarubicin is frequently used in chemotherapy against tumors. However, clinical use is severely limited by the development of progressive dose-dependent cardiomyopathy and acquired drug resistance. LY294002 is a commonly used pharmacologic inhibitor, which selectively inhibits the phosphoinositide 3-kinase-AKT nexus. The aim of this study was to investigate the combined inhibitory effect of LY294002 and pirarubicin on human osteosarcoma (OS) cells in vitro. The inhibitory effect of LY294002 plus pirarubicin on U2-OS and MG-63 OS cell proliferation, apoptosis, migration and invasion was investigated by cell proliferation, wound healing and Transwell invasion assays. The results revealed that LY294002 and pirarubicin synergistically induced apoptosis, and inhibited the growth, migration and invasion of OS cells. This indicates that LY294002 enhanced the effects of pirarubicin on OS in vitro. LY294002 combined with pirarubicin may thus be a future therapeutic strategy in OS.
\end{abstract}

\section{Introduction}

Osteosarcoma (OS) is the most common type of malignant tumor of the bone. Due to the rapid and aggressive nature of the disease, the standard treatment for OS was previously amputation of the affected limb; however, the cure rate associated with this method was $<10 \%$ and almost all patients succumbed to their disease within one year from initial diagnosis. Over the past three decades, with the development of effective chemotherapeutic agents, the five-year cumulative survival

Correspondence to: Dr Zhi Li Liu, Department of Orthopedics, First Affiliated Hospital of Nanchang University, 17 Yong Wai Zheng Street, Nanchang, Jiangxi 330006, P.R. China

E-mail: zgm7977@163.com

*Contributed equally

Key words: osteosarcoma, metastasis, pirarubicin, LY294002, synergistic action rate of primary OS patients has significantly improved to $60-90 \%$ (1-7). Chemotherapy is, thus, an important treatment for OS.

Pirarubicin, a member of the anthracycline family, is widely applied in the treatment of OS (8-12). However, its clinical use is severely limited by the development of progressive dose-dependent cardiomyopathy and acquired drug resistance (13). Adjunctive drug therapies occasionally reduce adverse reactions and drug resistance. Therefore, drugs that enhance the effect of pirarubicin against OS are being investigated.

The phosphoinositide 3-kinase (PI3K)/AKT signaling pathway is important in tumor cell differentiation, cell cycle, apoptosis, growth and metastasis $(8,9)$. Recent studies have shown that the PI3K/AKT signaling pathway is crucially involved in the resistance of cancer cells to chemotherapy $(14,15)$. Therefore, inhibition of the PI3K/AKT signaling pathway is under investigation as a potential target for cancer therapy. LY294002 is a commonly used pharmacological PI3K inhibitor, which acts on the ATP-binding site of the PI3K enzyme and thus selectively inhibits the PI3K-AKT nexus. Numerous studies have demonstrated that LY294002 enhances the chemosensitivity of various cancer types to a wide variety of drugs (16-19). However, whether LY294002 enhances the effects of pirarubicin on OS remains unclear.

In the present study, the effect of LY294002 on the sensitivity of OS cell lines to pirarubicin chemotherapy was investigated, in addition to the possible mechanisms underlying any such effect.

\section{Materials and methods}

Cell lines and cell culture. The U2-OS and MG-63 human OS cell lines were obtained from the American Type Culture Collection (Manassas, VA, USA). The cells were routinely cultured in Dulbecco's modified Eagle's medium (Hyclone, Waltham, MA, USA) supplemented with $10 \%$ fetal bovine serum (Sigma-Aldrich, St. Louis, MO, USA) in a humidified $37^{\circ} \mathrm{C}$ incubator containing $5 \% \mathrm{CO}_{2}$.

Cell growth assay. The cells were cultured in 96-well tissue culture plates at a density of 5,000 cells/well in minimum 
essential medium containing $10 \%$ fetal bovine serum and $2 \mathrm{mM}$ L-glutamine. Following overnight attachment, the medium was replaced and the U2-OS cells were incubated with various concentrations of LY294002 (5, 10, 20 and $40 \mu \mathrm{g} / \mathrm{ml}$; Sigma-Aldrich), pirarubicin (0.1, $0.2,0.4$ and $0.8 \mu \mathrm{g} / \mathrm{ml}$; Zhejiang Hisun Pharmaceutical Co., Ltd, Zhejiang, China) or the two drugs combined at different ratios (Pirarubicin:LY294002, 1:25, 1:50 and 1:100). For the MG-63 cells, the medium was replaced following overnight attachment, and the cells were incubated with various concentrations of LY294002 $(2.5,5,10$ and $20 \mu \mathrm{g} / \mathrm{ml})$, pirarubicin $(0.25,0.5$, 1 and $2 \mu \mathrm{g} / \mathrm{ml}$ ) or the two drugs combined at different ratios (Pirarubicin:LY294002, 1:5, 1:10 and 1:20). Following treatment for $24 \mathrm{~h}, \mathrm{MTT}$ assays (Promega Corporation, Madison, WI, USA) were conducted using a spectrophotometer (Thermo Fisher Scientific, Waltham, MA, USA) at $490 \mathrm{~nm}$ wavelength in triplicate. The ratio of inhibition by LY294002, pirarubicin or combination treatment at each concentration was calculated, and concentration-viability curves were fitted by Originpro 9.0 software (OriginLab Corporation, Northampton, MA, USA). The half maximal inhibitory concentration (IC50) values of LY294002, pirarubicin and the combination treatment were determined. All experiments were repeated three times over multiple days. The interaction between pirarubicin and LY294002 was assessed using the combination index (CI), calculated as follows: $\mathrm{CI}=\mathrm{C}_{\mathrm{AC}} / \mathrm{C}_{\mathrm{AS}}+\mathrm{C}_{\mathrm{BC}} / \mathrm{C}_{\mathrm{BS}}$. The $\mathrm{C}_{\mathrm{AC}}$ and $C_{B C}$ values indicate the concentrations of pirarubicin and LY294002, respectively, in a combined model when the inhibition ratio is $50 \%$. The $\mathrm{C}_{\mathrm{AS}}$ and $\mathrm{C}_{\mathrm{BS}}$ values indicate the concentrations of LY294002 and pirarubicin, respectively, at $50 \%$ inhibition in single drug treatment. When CI is $<0.95$, this signifies a synergistic effect between pirarubicin and LY294002. However, when CI is $>1.05$, this signifies an antagonizing effect between the two drugs. When CI is calculated to be between 0.95 and 1.05 , this indicates an additive effect between pirarubicin and LY294002.

Nuclear staining with 4,6-diamidino-2-phenylindole (DAPI). Following treatment with either LY294002 or pirarubicin alone, or the combination of the two, the cells were fixed with $3.7 \%$ paraformaldehyde (Sigma-Aldrich) in phosphate-buffered saline (PBS) for $8 \mathrm{~min}$ at room temperature. The fixed cells were washed with PBS and stained with DAPI (Sigma-Alrich) solution for $5 \mathrm{~min}$ at room temperature. The cells were then washed three more times with PBS and analyzed with a fluorescence microscope (Olympus Corporation, Tokyo, Japan). Jin's $\mathrm{Q}$-value, which is also used to assess the adjunctive therapy, was determined by the following equation: $\mathrm{Q}=\mathrm{E}(\mathrm{A}+\mathrm{B}) /$ $[\mathrm{EA}+(1-\mathrm{EA}) \mathrm{xB}]$. The $\mathrm{E}(\mathrm{A}+\mathrm{B})$ term indicates the apoptotic cell percentage at various drug concentrations when the two drugs were combined, while EA and EB signify the respective apoptotic percentages when LY294002 and pirarubicin were used alone. When $\mathrm{Q}$ is $<0.85$, this demonstrates an antagonizing effect between LY294002 and pirarubicin. When Q is $>1.15$, this reveals a synergistic effect between the two drugs. When the Q-value is calculated as between 0.85-1.15, this signifies an additive effect between LY294002 and pirarubicin.

Invasion assay. The invasiveness of the OS cells was measured with BD BioCoat ${ }^{\mathrm{TM}}$ BD Matrigel ${ }^{\mathrm{TM}}$ Invasion Chambers (BD
Bioscience, Franklin Lakes, NJ, USA) according to the manufacturer's instructions. The medium in the lower chamber contained $15 \%$ fetal bovine serum as a source of chemoattractant. The MG-63 cells were suspended in serum-free medium containing either $0.25 \mu \mathrm{g} / \mathrm{ml}$ pirarubicin, $5 \mu \mathrm{g} / \mathrm{ml}$ LY294002 or $0.25 \mu \mathrm{g} / \mathrm{ml}$ pirarubicin combined with $5 \mu \mathrm{g} / \mathrm{ml}$ LY294002. The U2-OS cells were suspended in serum-free medium containing either $0.2 \mu \mathrm{g} / \mathrm{ml}$ pirarubicin, $10 \mu \mathrm{g} / \mathrm{ml}$ LY294002 or $0.2 \mu \mathrm{g} / \mathrm{ml}$ pirarubicin combined with $10 \mu \mathrm{g} / \mathrm{ml}$ LY294002. The cells were added to the upper chambers simultaneously $\left(2 \times 10^{3}\right.$ cells in $\left.0.1 \mathrm{ml}\right)$. The cells passing through the Matrigel-coated membrane were stained with Diff-Quik (Sysmex, Kobe, Japan) and images were captured using a camera (Canon, Tokyo, Japan). Cell invasion was quantified by direct microscopic visualization (Olympus Corporation) and counting. The invaded cells were counted from five randomly selected fields under an inverted microscope. Three independent experiments were performed over multiple days.

Migration assay. Cell migration was assessed by determining the ability of the cells to move into a cellular space in a two-dimensional wound healing assay in vitro. In brief, the cells were cultured in six-well tissue culture plastic dishes at a density of $5 \times 10^{6}$ cells/well. The MG-63 cells were treated with $0.25 \mu \mathrm{g} / \mathrm{ml}$ pirarubicin, $5 \mu \mathrm{g} / \mathrm{ml} \mathrm{LY} 294002$ or $0.25 \mu \mathrm{g} / \mathrm{ml}$ pirarubicin combined $5 \mu \mathrm{g} / \mathrm{ml} \mathrm{LY} 294002$ for $24 \mathrm{~h}$. The U2-OS cells were treated with $0.2 \mu \mathrm{g} / \mathrm{ml}$ pirarubicin, $10 \mu \mathrm{g} / \mathrm{ml}$ LY294002 or $0.2 \mu \mathrm{g} / \mathrm{ml}$ pirarubicin combined with $10 \mu \mathrm{g} / \mathrm{ml}$ LY294002 for $24 \mathrm{~h}$. The cells were denuded by dragging a rubber policeman (Fisher Scientific, Hampton, NH, USA) through the center of the plate well. The culture plates were rinsed with PBS, then fresh quiescent medium, either alone or with $10 \%$ BSA, was added, and the cells were incubated at $37^{\circ} \mathrm{C}$ for $24 \mathrm{~h}$. Images of the cells were captured at 0 and $24 \mathrm{~h}$ and the migration distances were measured. The migration rate was calculated from five randomly selected fields under an inverted microscope. Three independent experiments were performed over multiple days.

Statistical Analysis: Data are expressed as the mean \pm standard deviation. The differences in proliferation, apopotosis, invasion and migration between groups were evaluated with one-way analysis of variance. $\mathrm{P}<0.05$ was considered to indicate a statistically significant difference. All analysis were performed with SPSS Version 19.0 (SPSS, Inc., Chicago, IL, USA).

\section{Results}

LY294002 enhances the inhibitory effect of pirarubicin on OS cell growth. The effect of LY294002, pirarubicin and the combination treatment on the growth of the U2-OS and MG-63 cell lines was examined by an MTT assay. The growth curves indicated that the MG-63 and U2-OS cells were sensitive to LY294002 and pirarubicin, and growth inhibition occurred in a dose-dependent manner (Fig. 1A, B, D and E). The pirarubicin IC50 values for MG-63 and U2-OS cells were 3.61 and $0.47 \mu \mathrm{g} / \mathrm{ml}$, respectively. The LY294002 IC50 values for U2-OS and MG-63 cells were 19.71 and $17.36 \mu \mathrm{g} / \mathrm{ml}$ respectively. When these two reagents were administered to U2-OS cells 
A

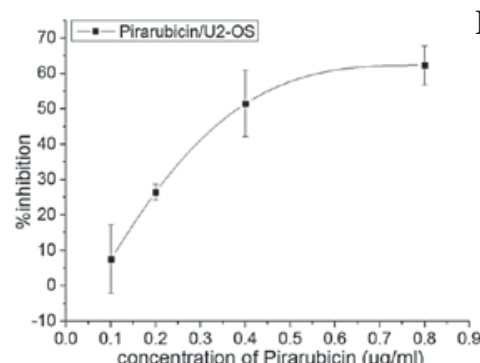

D

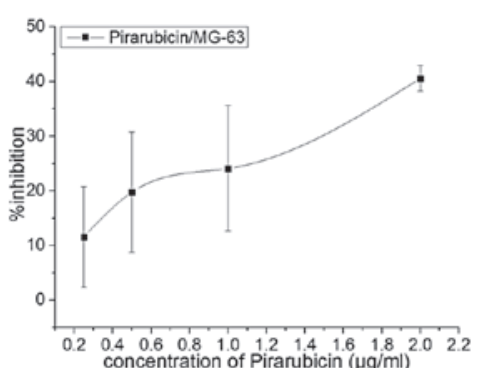

B

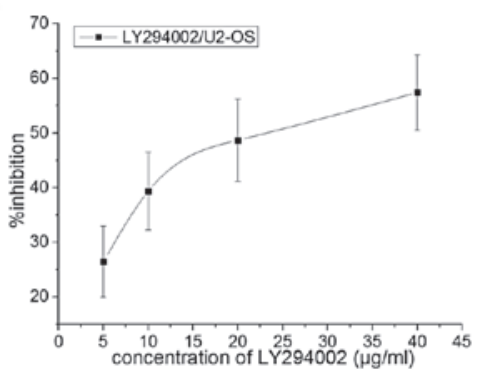

$\mathbf{E}$

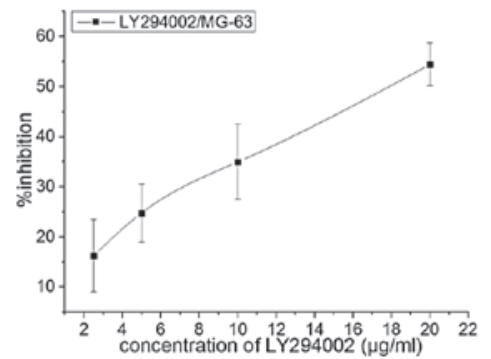



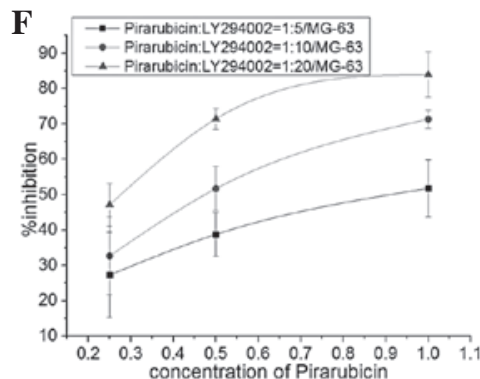

Figure 1. Effect of LY294002 and pirarubicin on osteosarcoma (OS) cell growth. LY294002 and pirarubicin inhibit the growth of U2-OS and MG-63 OS cells in a dose-dependent manner (A, B, D and E). Administration of the two reagents in combination in different proportions exerted a synergistic antitumor effect $(\mathrm{C}$ and $\mathrm{F}$; combination index<0.95).

A



D

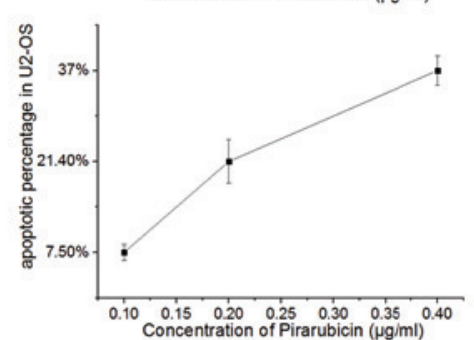

G

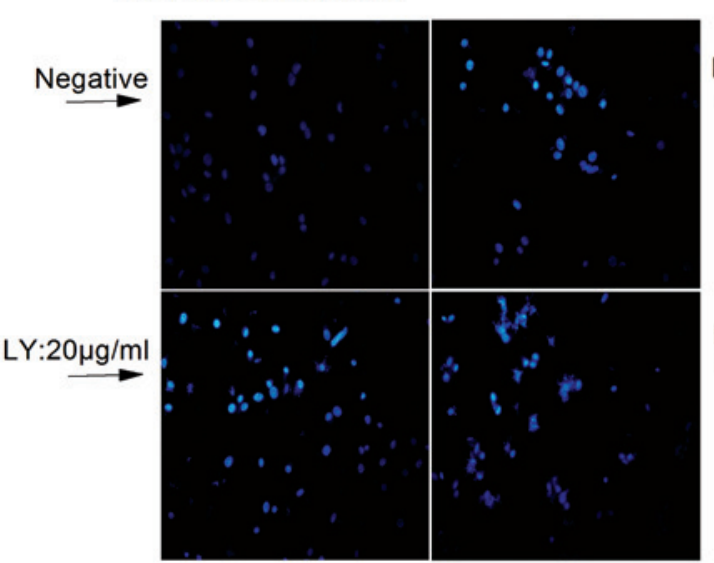

MG-63
B

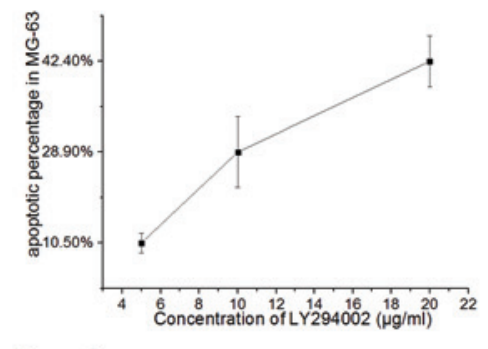

E

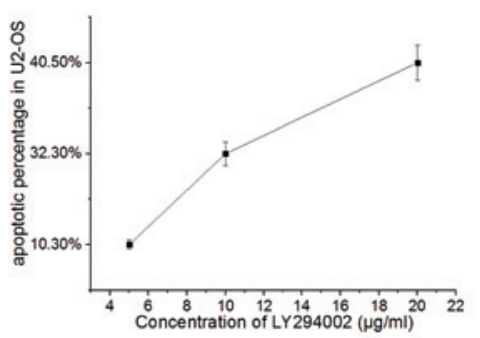

H
C

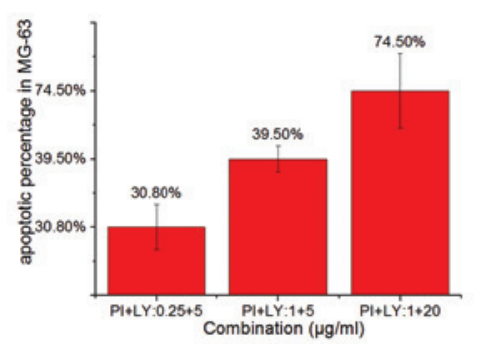

F

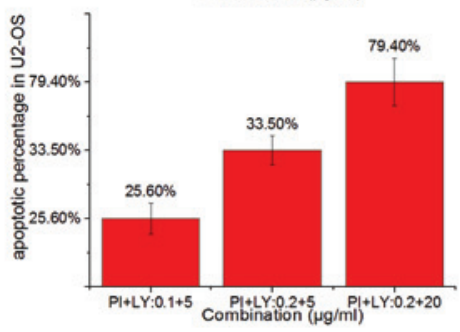

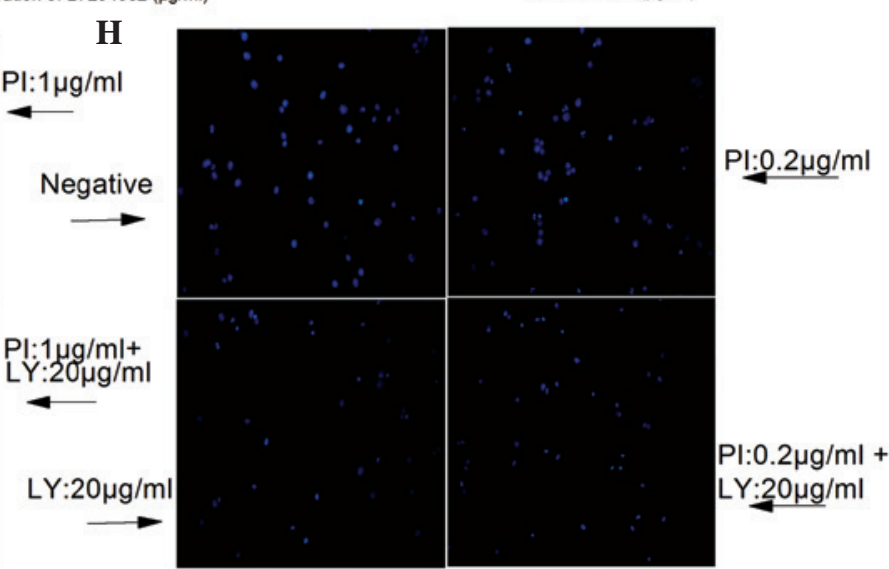

U2-OS

Figure 2. Effect of LY and PI on OS cell apoptosis. (A) and (D) Apoptotic percentage of MG-63 (A) and U2-OS (D) cells with varying concentrations of PI. (B) and (E) Apoptotic percentage of MG-63 (B) and U2-OS (E) cells with varying concentrations of LY. (C) and (F) Apoptotic percentage of MG-63 (C) and U2-OS (F) cells with different combinations of LY and PI. (G) and (H) Effect of LY, PI or combination on MG-63 (G) and U2-OS (H) cells. Apoptosis was examined by nuclear staining with DAPI. LY, LY294002; PI, pirarubicin; OS, osteosarcoma. 
A

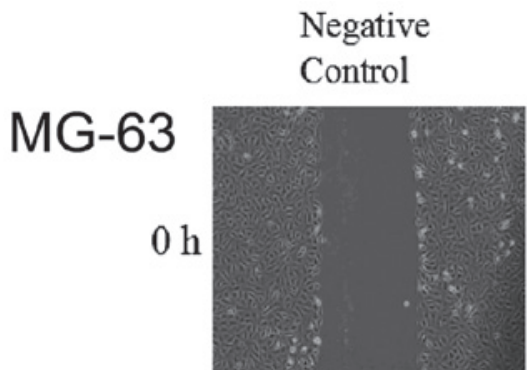

$0.25 \mu \mathrm{g} / \mathrm{ml}$ Pirarubicin
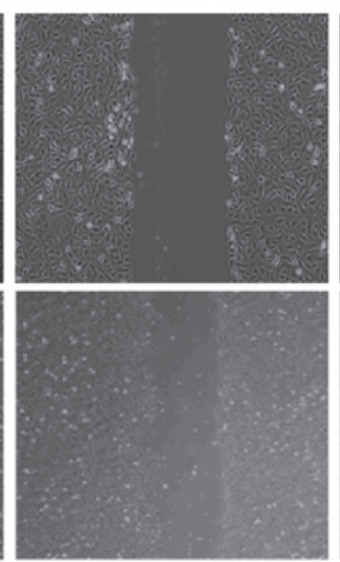

B

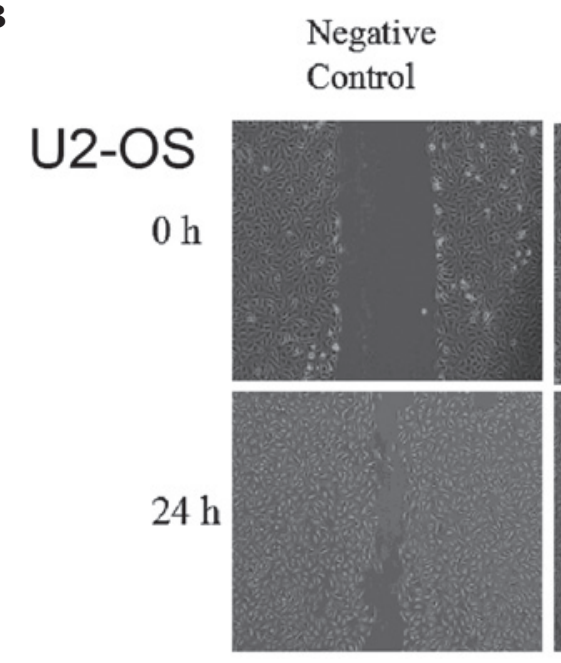

$0.2 \mu \mathrm{g} / \mathrm{ml}$

Pirarubicin
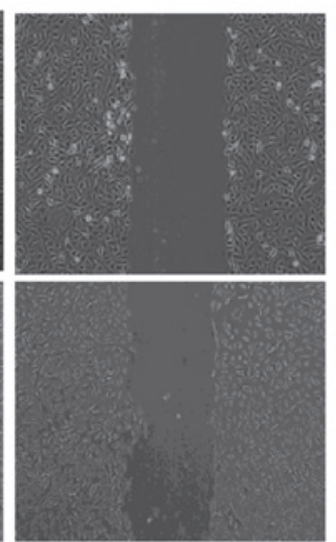

$5 \mu \mathrm{g} / \mathrm{ml}$ LY294002
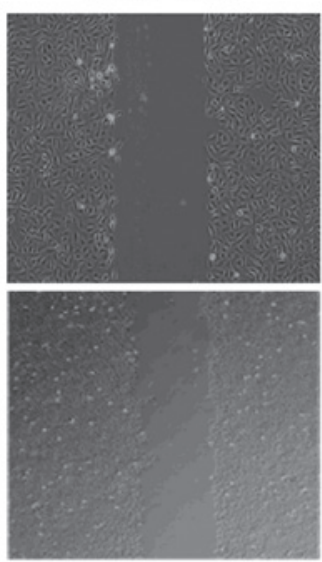

$10 \mu \mathrm{g} / \mathrm{ml}$ LY294002
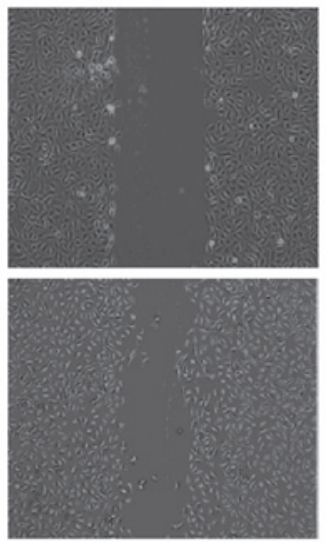

combined
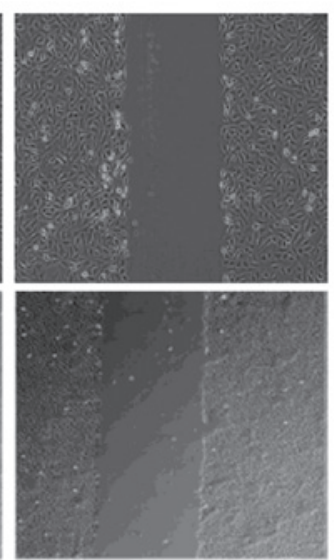

combined

Figure 3. Effect of LY294002 and pirarubicin on (A) MG-63 and (B) U2-OS osteosarcoma (OS) cell migration. The migration rate of OS cells treated with LY294002 plus pirarubicin was significantly lower than those treated by LY294002 or pirarubicin alone.

simultaneously at 1:25, 1:50 and 1:100 proportions (Fig. 1C), the IC50 values were $6.54,9.15$ and $11.28 \mu \mathrm{g} / \mathrm{ml}$, respectively. All CI values at 1:25, 1:50 and 1:100 proportions were $<0.95$ $(0.74,0.87$ and 0.81 respectively). When the two reagents were administered to MG-63 cells at 1:5, 1:10 and 1:20 proportions (Fig. 1F), the CI values were all $<0.95(0.54,0.39$ and 0.37 respectively). These results indicate a synergistic antitumor effect between LY294002 and pirarubicin.

LY294002 synergistically enhances pirarubicin-induced OS cell apoptosis. Nuclear staining with DAPI was used to examine the mechanism of the drug-induced promotion of cell apoptosis by LY294002, pirarubicin and the combination treatment. The results revealed that U2-OS and MG-63 cell apoptosis occurred in a dose-dependent manner, regardless of whether cells were treated with LY294002, pirarubicin or the combination of the two (Fig. 2A-F). However, when these two reagents were applied to U2-OS and MG-63 cells together, the apoptotic percentage was increased (Fig. $2 \mathrm{G}$ and $\mathrm{H}$ ).

Inhibitory effect of LY294002 and pirarubicin, administered separately and in combination, on OS cell migration. To examine the inhibitory effect of LY294002 and pirarubicin on
OS cell migration, wound-healing assays were performed. The cells were treated with LY294002 and pirarubicin, separately and combined, for $24 \mathrm{~h}$ in a wound healing system. The results revealed that the migration rate of cells treated with LY294002 and pirarubicin together was significantly lower than those treated by either LY294002 or pirarubicin alone (Fig. 3). The results suggest that LY294002 enhanced the inhibitory effect of pirarubicin on OS cell migration in vitro.

Inhibitory effects of LY294002, pirarubicin and combination treatment on OS cell invasion. To examine the inhibitory effect of LY294002 and pirarubicin on OS cell invasion, Transwell invasion assays were performed. The results demonstrated that the number of transmembranous cells following combined LY294002 and pirarubicin treatment was significantly lower than those treated by LY294002 and pirarubicin separately (Fig. 4). These data indicate that LY294002 enhanced the inhibitory effect of pirarubicin on OS cell invasion in vitro.

\section{Discussion}

OS is one of the most common primary malignant bone tumors in children and adolescents. With the advent of 
A
Negative
Control

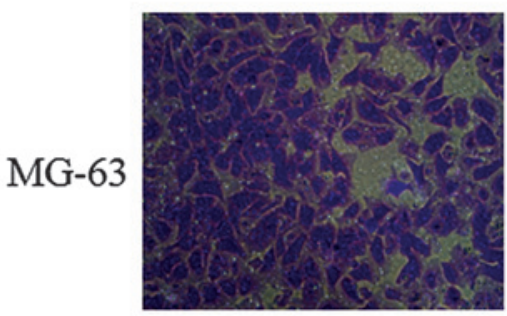

B

$$
\begin{aligned}
& \text { Negative } \\
& \text { Control }
\end{aligned}
$$

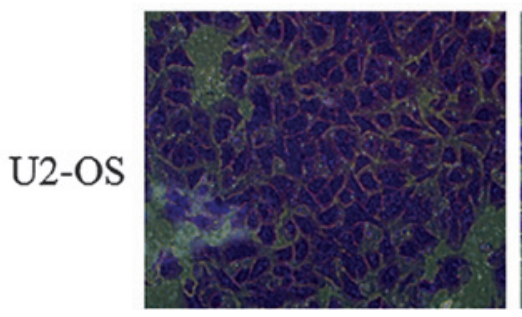

$0.25 \mu \mathrm{g} / \mathrm{ml}$ Pirarubicin

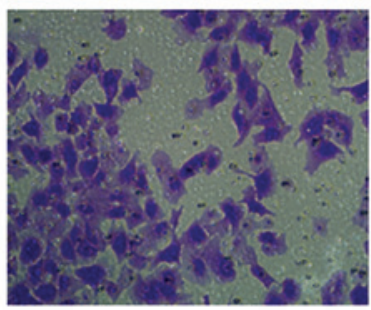

$0.2 \mu \mathrm{g} / \mathrm{ml}$ Pirarubicin

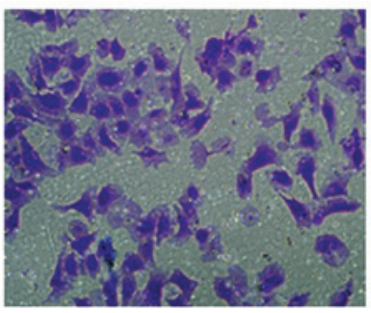

$5 \mu \mathrm{g} / \mathrm{ml}$ LY294002

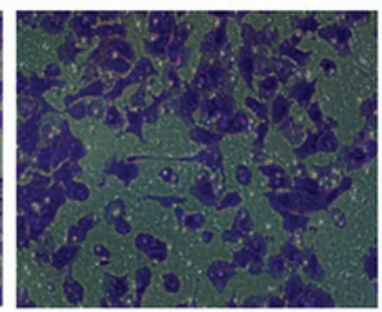

$10 \mu \mathrm{g} / \mathrm{ml}$ LY294002

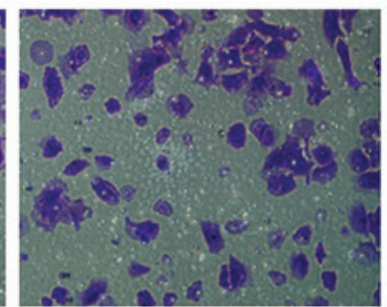

combined

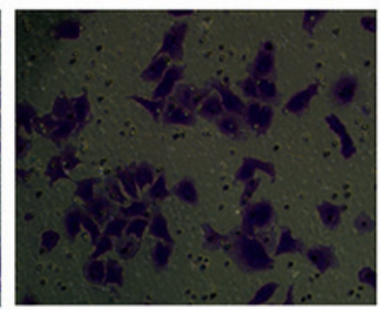

combined

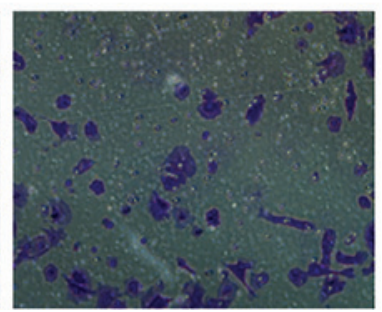

Figure 4. Effect of LY294002 and pirarubicin on (A) MG-63 and (B) U2-OS osteosarcoma (OS) cell invasion stained with Diff-Quick. The number of transmembranous cells in cells treated with LY294002 plus pirarubicin was significantly lower than those treated by LY294002 or pirarubicin separately. Magnification, x400.

effective chemotherapy, the five-year survival rate for patients treated with intensive multidrug chemotherapy and aggressive local control has been reported at 55-80\% (3,20,21). However, chemotherapy may fail to eliminate all osteosarcoma cells due to intrinsic or acquired drug resistance, which is the most frequent cause of tumor recurrence and results in a poor clinical outcome (22). Therefore, optimizing treatment strategies to improve the effect of chemotherapy is the predominant focus in OS treatment.

LY294002, a selective inhibitor of PI3K, is the first artificially synthesized small molecular inhibitor of PI3K $\alpha / \delta / \beta$. LY294002 acts on the ATP-binding site of PI3K and exhibits greater stability than wortmannin in solution (23). LY294002 inhibits cell proliferation and induces apoptosis by downregulating $\mathrm{AKT} /$ protein kinase $\mathrm{B}$ activation. A study by Semba et al (24) demonstrated that LY294002 inhibited cell growth and induced apoptosis by reducing AKT phosphorylation (Ser473) in colon cancer cell lines. Other studies have observed that LY294002 inhibited cell growth and induced apoptosis in various other cancer types $(25,26)$. Due to its versatile effects, LY294002 is a leading compound in the development of more effective and tolerable reagents in cancer treatment. For example, Xie et al (16) revealed that LY294002 enhanced the chemosensitivity of human gastric cancer to vincristine. In the present study, LY294002 was found to induce OS cell apoptosis and inhibit growth in a dose-dependent manner.

Pirarubicin, a member of the anthracycline family, is widely applied in OS treatment. However, the acquired resistance of cancer cells to pirarubicin has become a major obstacle in cancer therapy. Meesungnoen et al (27) suggested that P-glycoprotein (P-gp)-mediated Tetrahydropyranyl (THP) efflux and THP accumulation in acidic organelles confers an advantage for multidrug-resistant (MDR) cells in surviving prolonged exposure to cytotoxic agents and induces a high degree of resistance. The transmembrane transport pump P-gp causes the efflux of chemotherapeutic agents from cells and is an important system that secures MDR in neoplastic cells. Liang et al (28) found that inhibition of the PI3K/AKT signaling pathway by the specific inhibitor LY294002 or by AKT small interfering RNA resulted in reduced multidrug resistance of SGC7901 cells through downregulation of P-gp. Imai et al (29) reported that LY294002 inhibited P-gp, breast cancer resistance protein and multidrug resistance-associated protein 1 , three major ATP-binding cassette transporters that are highly expressed in cancer cells and cause multidrug resistance. Therefore, LY294002 was hypothesized to enhance the effects of pirarubicin on OS, which was confirmed in the present study. The results revealed that when pirarubicin was applied in combination with LY294002 in differential proportions, a synergistic antitumor effect between the two drugs was achieved, with $\mathrm{Q}>1.15$ and $\mathrm{CI}<0.95$. These data suggest that LY294002 enhanced pirarubicin-induced OS cell apoptosis and inhibited cell proliferation. In addition, the inhibitory effects of LY294002 and pirarubicin, administered separately and in combination, on OS cell migration and invasion were evaluated by wound healing and Transwell invasion assays. The results revealed that the number of transmembranous cells and the migration rate were significantly lower in the cells treated with LY294002 and pirarubicin than those treated with either LY294002 or pirarubicin alone.

In conclusion, the results of the present study indicate that a synergistic in vitro anticancer effect in OS exists between LY294002 and pirarubicin. This suggests that LY294002 combined with pirarubicin may be a strategy in OS treatment. However, the LY294002 and pirarubicin combination was only administered in vitro. In addition, the mechanism involved in 
the synergistic effect between pirarubicin and LY294002 remains unknown. Thus, further studies are required to elucidate the effect of LY294002 combined with pirarubicin on OS growth in vivo and to determine the potential underlying molecular mechanisms.

\section{Acknowledgements}

The present study was supported by the National Natural Science Foundation of China (grant no. 81260400) and the Natural Science Fundation of Jiangxi Province (grant no. 20114BAB205093).

\section{References}

1. Lewis VO: What's new in musculoskeletal oncology. J Bone Joint Surg Am 89: 1399-1407, 2007.

2. Iwamoto Y, Tanaka K, Isu K, et al: Multiinstitutional phase II study of neoadjuvant chemotherapy for osteosarcoma (NECO study) in Japan: NECO-93J and NECO-95J. J Orthop Sci 14: 397-404, 2009.

3. Meyers PA, Schwartz CL, Krailo M, et al: Osteosarcoma: a randomized, prospective trial of the addition of ifosfamide and/or muramyl tripeptide to cisplatin, doxorubicin, and high-dose methotrexate. J Clin Oncol 23: 2004-2011, 2005.

4. Lee JW, Kim H, Kang HJ, et al: Clinical characteristics and treatment results of pediatric osteosarcoma: the role of high dose chemotherapy with autologous stem cell transplantation. Cancer Res Treat 40: 172-177, 2008.

5. Cho Y, Jung GH, Chung SH, et al: Long-term survivals of stage IIB osteosarcoma: a 20-year experience in a single institution. Clin Orthop Surg 3: 48-54, 2011.

6. Tsuchiya H, Tomita K, Mori Y, et al: Caffeine-assisted chemotherapy and minimized tumor excision for nonmetastatic osteosarcoma. Anticancer Res 18: 657-666, 1998.

7. Bölling T, Schüller P, Distelmaier B, et al: Perioperative high-dose rate brachytherapy using a bendy applicator (flab): treatment results of 74 patients. Anticancer Res 28: 3885-3890, 2008.

8. Qi WX, He AN, Tang LN, et al: Evaluation of pirarubicin-cisplatin chemotherapy in the treatment for refractory and recurrent high-grade osteosarcoma: experience of a single institute. Med Oncol 29: 2229-2233, 2012.

9. Futani H, Fukunaga S, Tsukamoto Y, et al: Small cell osteosarcoma successfully treated by high-dose ifosfamide and methotrexate, combined with carboplatin and pirarubicin. Anticancer Res 32: 965-971, 2012.

10. Zhao H, Yao Y, Wang Z, et al: Therapeutic effect of pirarubicin-based chemotherapy for osteosarcoma patients with lung metastasis. J Chemother 22: 119-124, 2010.

11. Shinozaki T, Watanabe H, Yanagawa T, et al: Pirarubicin-based versus doxorubicin-based osteosarcoma chemotherapy. Ann Pharmacother 36: 996-999, 2002.

12. He A, Qi W, Huang Y, et al: Comparison of pirarubicin-based versus gemcitabine-docetaxel chemotherapy for relapsed and refractory osteosarcoma: a single institution experience. Int J Clin Oncol 18: 498-505, 2013.
13. Niitsu N, Yamazaki J, Nakayama M and Umeda M: Pirarubicininduced myocardial damage in elderly patients with non-Hodgkin's lymphoma. Nihon Ronen Igakkai Zasshi 35: 358-362, 1998 (In Japanese)

14. García MG, Alaniz LD, Cordo Russo RI, et al: PI3K/Akt inhibition modulates multidrug resistance and activates NF-kappaB in murine lymphoma cell lines. Leuk Res 33: 288-296, 2009.

15. Yu HG, Ai YW, Yu LL, et al: Phosphoinositide 3-kinase/Akt pathway plays an important role in chemoresistance of gastric cancer cells against etoposide and doxorubicin induced cell death. Int J Cancer 122: 433-443, 2008.

16. Xie X, Tang B, Zhou J, et al: Inhibition of the PI3K/Akt pathway increases the chemosensitivity of gastric cancer to vincristine. Oncol Rep 30: 773-782, 2013.

17. Wu D, Tao J, Xu B, et al: Phosphatidylinositol 3-kinase inhibitor LY294002 suppresses proliferation and sensitizes doxorubicin chemotherapy in bladder cancer cells. Urol Int 87: 105-113, 2011.

18. Kwon O, Kim KA, Kim SO, et al: NF-kappaB inhibition increases chemosensitivity to trichostatin A-induced cell death of Ki-Ras-transformed human prostate epithelial cells. Carcinogenesis 27: 2258-2268, 2006.

19. Fu ZY, Lv JH, Ma CY, et al: Tissue inhibitor of metalloproteinase-1 decreased chemosensitivity of MDA-435 breast cancer cells to chemotherapeutic drugs through the PI3K/AKT/NF- $\mathrm{BB}$ pathway. Biomed Pharmacother 65: 163-167, 2011.

20. Bacci G, Forni C, Longhi A, et al: Local recurrence and local control of non-metastatic osteosarcoma of the extremities: a 27-year experience in a single institution. J Surg Oncol 96: 118-123, 2007.

21. Jawad MU,Cheung MC, Clarke J, et al: Osteosarcoma: improvement in survival limited to high-grade patients only. J Cancer Res Clin Oncol 137: 597-607, 2011.

22. Ferrari S, Smeland S, Mercuri M, et al; Italian and Scandinavian Sarcoma Groups: Neoadjuvant chemotherapy with high-dose Ifosfamide, high-dose methotrexate, cisplatin, and doxorubicin for patients with localized osteosarcoma of the extremity: a joint study by the Italian and Scandinavian Sarcoma Groups. J Clin Oncol 23: 8845-8852, 2005.

23. Wu D, Tao J, Xu B, et al: Phosphatidylinositol 3-kinase inhibitor LY294002 suppresses proliferation and sensitizes doxorubicin chemotherapy in bladder cancer cells. Urol Int 87: 105-113, 2011.

24. Semba S, Itoh N, Ito M, et al: The in vitro and in vivo effects of 2-(4-morpholinyl)-8-phenyl-chromone (LY294002), a specific inhibitor of phosphatidylinositol 3'-kinase, in human colon cancer cells. Clin Cancer Res 8: 1957-1963, 2002.

25. Hu L, Zaloudek C, Mills GB, et al: In vivo and in vitro ovarian carcinoma growth inhibition by a phosphatidylinositol 3-kinase inhibitor (LY294002). Clin Cancer Res 6: 880-886, 2000.

26. Chen Z, Yang L, Liu Y, et al: LY294002 and Rapamycin promote coxsackievirus-induced cytopathic effect and apoptosis via inhibition of PI3K/AKT/mTOR signaling pathway. Mol Cell Biochem 385: 169-177, 2014.

27. Meesungnoen J, Jay-Gerin JP and Mankhetkorn S: Relation between MDR1 mRNA levels, resistance factor, and the efficiency of P-glycoprotein-mediated efflux of pirarubicin in multidrug-resistant K562 sublines. Can J Physiol Pharmacol 80: 1054-1063, 2002

28. Liang J, Ge F, Guo C, et al: Inhibition of PI3K/Akt partially leads to the inhibition of $\operatorname{Pr} P(C)$-induced drug resistance in gastric cancer cells. FEBS J 276: 685-694, 2009.

29. Imai Y, Yamagishi H, Ono Y and Ueda Y: Versatile inhibitory effects of the flavonoid-derived PI3K/Akt inhibitor, LY294002, on ATP-binding cassette transporters that characterize stem cells. Clin Transl Med 1: 24, 2012. 